\title{
STUDY OF THERMAL AND MECHANICAL PROPERTIES OF FIBER- GLASS MULTI-WALL CARBON NANOTUBE/EPOXY
}

\author{
Luay Hashem Abbud, ${ }^{\mathrm{a}, \text {, }}$, Hyder H. Balla ${ }^{\mathrm{c}}$, Ammar F. Abdulwahid ${ }^{\mathrm{d}}$, Zaid Sttar Karim ${ }^{\mathrm{d}}$ \\ a Air conditioning and Refrigeration Technical Engineering Department, Al-Mustaqbal University College, Babylon, Iraq \\ ${ }^{b}$ Department of Aeronautics Technical, Al-Furat Al-Awsat Technical University, Technical Institute of Najaf, Iraq \\ ${ }^{c}$ Al-Furat Al-Awsat Technical University, Technical Institute of Najaf, Iraq \\ ${ }^{d}$ Department of Mechanical engineering, University of Kufa, Najaf, Iraq
}

\begin{abstract}
This project aims at determining both numerical and experimental to some thermal properties and its thermal expansion coefficient, thermal conductivity and mechanical properties of reinforcement of fiber glass woven with matrix of multi wall carbon nanotube MWCNT / epoxy composite. First, this powder is known to have a very good thermal properties. So, the nanopartical combined with resin has poor thermal properties. Secondly, the development a complete solution for the manufacturing of multi wall carbon nanotube /epoxy composites different volume fraction from $1 \%$ to $10 \%$ with increment of $2 \%$ to compare the result of finite element method by using ANSYS program with experimental results to determine the mechanical and thermal properties for nanocomposite materials. The finite element by using ANSYS is good agreement with experimental data for different volume fraction. The thermal conductivity of the nanocomposite materials increases with increasing in the volume fraction concentration thermal expansion coefficient reduces with increasing in the volume fraction concentrations. The increment nano particle concentration effect on the mechanical properties is accomplished the best results.
\end{abstract}

Keywords: $M W C N T$, Numerical analysis, Thermal conductivity, volume fraction, ANSYS Program

\section{INTRODUCTION}

In this research, fiber glass with multi wall carbon nanotube MWCNT and epoxy was used to fill the gaps of previous research by reducing the weight and the cost of composite structures, especially those are used against light and other structures such as vehicles, aircraft and some applications. Although the advantages are provided by the composites materials in terms of the hardness and light weight resulting from the low density. The composite has less effect which is considered as one of its defects. fragmentation and cracking, which are leading to heavy damage in the tile nanocomposite that is injured by munitions, so that, there is not only damage to the area of injury, but rather to the surface of the board that has a larger area of spreading cracks, leaving people prone to injury, and because of this, the research was conducted to develop composite materials characteristics that do not allow the spread of cracks and prevent fragmentation at the exposure to diverse ammunition injuries. So, Multi wall carbon nanotubes with epoxy are among the fibers glass that covers even gaps were utilized in this work. Ahmad and Iqbal (2017) in this study the analysis of the thermal transfer phenomenon by using of Cattaneo-Christov heat flux model, through which the time of thermal relaxation is visualized and the fluid temperature is related to thermal relaxation.

The role of fiber glass is to prevent the cracks in addition to the composite fragmentation through the process of fragmentation. The volume of fiber portion and how it has an impact on the flexural properties is studied by Uleiwi (2007). He shows that the flexural properties of the composite have several strata. The first stratum has fortified glass fiber and the second stratum is strengthened with Kevlar fiber that has been explored tentatively and it is clarified that the strain pressure diminishes the expansion in the volume of fiber part of the lower stratum which consists of glass fiber, whereas its increments are with the expansion of Kevlar volume division of the upper stratum.

Tacir et al. (2006) studied the strengthening impact of glass filaments on the break obstruction and flexural properties of acrylic saps. In this examination, a huge contrasts were found in the flexural properties of the specimen. The infusion shaped is quintessential lower flexural features for the fiber which is fortified polymers than injecting formed composite materials. The microwave-shaped and the microwave-formed composites have different flexural features. The former is lower than the latter. It is found that a break opposition is fundamentally high in the injecting formed than in the injecting shaped composites, while the crack obstruction has the higher level in the microwave formed than in the microwave-shaped composites. Wen et al. (1998) conducted probes composite sandwich boards comprising of woven E-glass/polyester overlays and froth center and furthermore the expansion in the disappointment heap of the mixed sandwich underneath effect stacking when contrasted with static burden space. It is credited that the aggregate raises to the upgraded properties and frostiness of the glass/polyester face sheet and foam center at strain rates which are high just as the inactivity of the shot and sandwich plat of the composite. Structures of steady materials and sandwich plate have assorted and innovatively intriguing implementation. Those incorporate the expanded utilization of composite overlays in aviation and car designing. Habib et al. (2017) investigated numerical and experimentally used Nanofluid of different nanoparticles materials copper, $\mathrm{Al}_{2} \mathrm{O}_{3}$ and $\mathrm{TiO} 2$ with water as a base fluid. It was found that the thermal conductivity of copper is higher than other materials.

Buket (2002) investigates reconnaissance investigations to decide properties of adjustments adhesive Fiber - entrenched E-glass/epoxy components. Different mechanistic features and quality were decided

* Correspondent author. Email: luayhashem@yahoo.com 
hesitantly. The overlays fabricated had various directions of the Fibers. The parametric examination was piloted thinking about the geometry of the Fiber direction and discontent features for the stick fixed Fiber strengthened covered composites were broke down. A relationship between exploratory qualities with fewer elements integrated is carried out. It is shown that the utilize direction and the geometry of the components are vital if it has to be an amount of fixed Fiber fortified covered composites. Carbon nanotubes (CNT) are firstly revealed by Iijima (1991). Nandeppanavara and Shakunthala (2017) in this paper used carbon nanotube to study the effect of buoyancy on heat transfer and fluid flow. It was noticed that buoyancy has a positive effect on cooling the system. From that point forward numerous investigations have been accounted for with respect to their assembling, constructions, and features. Ajayan and Ebbesen (1997) and Ebbesen and Ajayan (1992) revealed about the production of CNT in huge rates, and furthermore expressed that the most straightforward approach to comprehend the construction of CNT is to reflect it as a stratum with twodimension of a graphite sheet. There are essentially two sorts of CNT: single divider nanotubes (SWNT) and multi divider nanotubes (MWNT), comprising of at least two concentric round and hollow shells of graphite pieces. Tashkandi and Aydi (2018) in this study, heat transfer was calculated using CNT and $\mathrm{Al} 2 \mathrm{O} 3$ with water and a different volume fraction. It was found that the increase of Rayleigh number increases the volume fraction and increases the heat transfer.

Designing and fabrication of composite plate specimens for different volume fractions of fibers glass are woven. A multi-wall carbon nanotube MWCNT/epoxy and test specimens to obtain the mechanical properties, thermal conductivity, thermal expansion coefficient and mechanical properties of experimental and numerical that uses finite element method ANSYS program. The use of new combinations of materials: resin Multi wall carbon nanotube/epoxy with reinforcement a woven fiber glass reduce weight and cost and increase strength.

\section{EXPERIMENTAL SETUP}

It is notable that laminate composites materials are comprised of layers at any rate of two distinct materials that are reinforced together. Overlay is utilized to join the best parts of the constituent layers so as to accomplish progressively helpful materials. Both thermal and mechanical properties for the composites are utilized, particularly the composite materials that are produced using fiber glass and multi - wall carbon nanotube (MWCNT) and for fiber geometrics that is woven. The grid utilized is epoxy resin. Fiber and MWCNT fortifications and epoxy resin, the fiber composite material overlays are manufactured essentially from two primary constituents, which are the fiber glass and MWCNT support, and the unsaturated resin. Remember that the between employ layer, or the glue layer between any two progressive layers, is simply the gum with some extra thickness for recognizing this exchange layer. The accompanying shows the chemical and physical compound particulars of these two constituents.

\subsection{Multi-Wall carbon nanotube}

The specific MWCNT was used in all spacemen constructions MWCNT. The specific MWCNT properties are shown in Table 1.

Table 1 The Physical Properties of multi-wall carbon nanotube (MWCNT)

\begin{tabular}{c|ccccc}
\hline $\begin{array}{c}\text { Density } \\
\mathrm{Kg} / \mathrm{m}^{3}\end{array}$ & $\begin{array}{c}\text { Thermal } \\
\text { conductivity } \\
W / m K\end{array}$ & $\begin{array}{c}\text { Electrical } \\
\text { Conductivity } \\
\text { S/cm }\end{array}$ & $\begin{array}{c}\text { Length } \\
\mu m\end{array}$ & $\begin{array}{c}\text { Diameter } \\
n m\end{array}$ & Purity \\
\hline $\mathbf{2 1 0 0}$ & 3000 & 100 & $10-30$ & $\begin{array}{c}\text { Inner: } 5-10 \\
\text { Outer: } 10-30\end{array}$ & $90 \%$ \\
\hline
\end{tabular}

\subsection{Fiberglass}

Glass fiber is a material produced using incredibly fine fibers of glass. It is utilized as a fortifying operator for some polymer items. This composite material, regularly known as Fiber Reinforced Polymer (FRP) is all the more prominently alluded to as fiberglass properties are shown in Table 2.

Table 2 The Mechanical and Thermal properties of Fiber glass

\begin{tabular}{|c|c|c|c|c|c|}
\hline $\begin{array}{c}\text { Tensile } \\
\text { strengt } \\
\quad h \\
M P a\end{array}$ & $\begin{array}{c}\text { Elastic } \\
\text { Modulu } \\
\text { S } \\
G P a\end{array}$ & $\begin{array}{c}\text { Densit } \\
y \\
\mathrm{Kg} / \mathrm{m}^{3}\end{array}$ & $\begin{array}{c}\text { Thermal } \\
\text { conductivit } \\
y \mathrm{~W} / \mathrm{mK}\end{array}$ & $\begin{array}{c}\text { Thermal } \\
\text { Expansion } \\
\text { coefficient } \\
1 /{ }^{\circ} \mathrm{C}\end{array}$ & $\begin{array}{c}\text { Poisson' }^{\prime} \\
\text { s Ratio }\end{array}$ \\
\hline 2050 & 85 & 2600 & 1.35 & $5.1 \times 10^{-6}$ & 0.23 \\
\hline
\end{tabular}

\subsection{Epoxy Resin}

Epoxy resins are used extensively in composite materials, due to their commercial availability and versatility, formerly known as a low consistency, fluid epoxy resin produced from epichlorohydrin and Bisphenol F. This tar contains no diluents or modifiers. The perfect auxiliary recipe. As appeared in Table 3 Mechanical and Thermal properties.

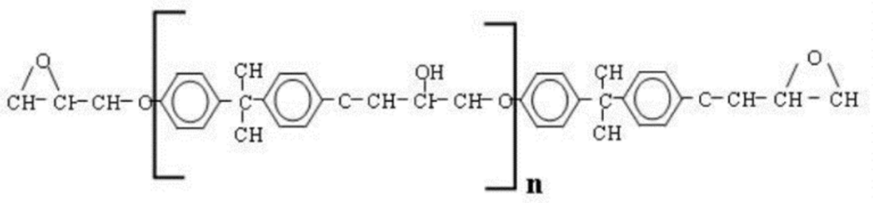

Fig. 1 Structure of epoxy resin

Table 3: Mechanical and Thermal Properties of Epoxy resin

\begin{tabular}{c|ccccc}
\hline $\begin{array}{c}\text { Tensile } \\
\text { strength }\end{array}$ & $\begin{array}{c}\text { Elastic } \\
\text { Modulus } \\
\mathrm{GPa} a\end{array}$ & $\begin{array}{c}\text { Density } \\
\mathrm{Kg} / \mathrm{m}^{3}\end{array}$ & $\begin{array}{c}\text { Thermal } \\
\text { conductivity } \\
\mathrm{W} / \mathrm{mK}\end{array}$ & $\begin{array}{c}\text { Thermal } \\
\text { Expansion } \\
\text { coefficient } \\
1 / C\end{array}$ & $\begin{array}{c}\text { Poisson's } \\
\text { Ratio }\end{array}$ \\
\hline $\mathbf{5 2 . 2}$ & 3.5 & 1309.44 & 0.1525 & $5.7610^{-5}$ & - \\
\hline
\end{tabular}

\subsection{Specimens Preparation}

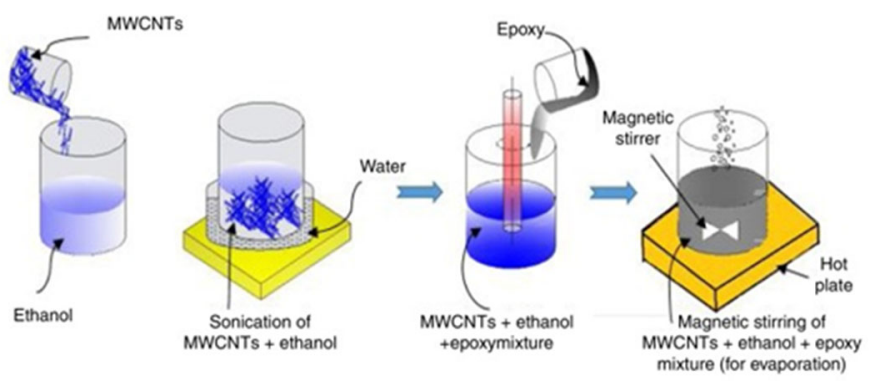

Fig. 2 Technical mixtures MWCNT / Epoxy

There are two gathering of tests formed, framework tests and layered fiber, powder composite plate tests. The lattice tests were made to confirm the mechanical properties of the matrix, particularly in the light of the fact that the natural conditions and procedure of embellishment are significant parameters influencing the properties, in light of this there are no offered properties that can be utilized straightforwardly, so the tensile test was finished. The way that component plate tests are done is by utilizing various strata and grid material to check the mechanistic and thermal properties. The composite plate band specimen utilized in that examination was created through the fiberglass/carbon nanotube mold 
employs, and balanced lay-ups are set up by utilizing a $25 * 20 \mathrm{~cm}$ glass sheet that is open form with an $\mathrm{x}$-ray photograph sheet painted with wax film to maintain a strategic distance from scraped area and inshore straightening. The decay applies a shade "discharge" material to the form as the initial phase in making any open shape item. Supposing that this material is absent, the element is going to for all time attach to the shape surface. Besides, a pre-estimated unsaturated matrix blended with MWCNT was then altogether combined, and to guarantee total air and dampness evacuation, the base surface has to be covered totally, particularly at the sides as appeared in Fig. 2. The third step was utilization of fiber in the blend (MWCNT + epoxy) stratum; those knitted strata are placed in the (MWCNT + epoxy) layer and utilizing brushes and rollers the fiber layer would be soaked by blending, at that point another fiber sheet would be put down and the procedure rehashed until the last layer, at that point the $\mathrm{x}$ - ray photograph sheet covered with wax would cover the composite, moving over all layers to guarantee total air evacuation. Composite plates were produced using fiber glass, MWCNT and epoxy to check the mechanical and thermal properties.

\subsection{Tensile and Compression tests of the composite}

To make this examination significant, one has to take in concern the stress - strain conduct of the fiber glass and MWCNT/Epoxy for multidirectional knitted fiber. By using a standard testing machine (Instron model 3366), users finish working on the tensile and compression test devices. Tensile tests are done through the standard (ASTM D 3039/D 3039 M-95a). Features of tensile are decided through the standard technique in the in-plane tensile features of polymer grid composite elements that strengthened through high modulus fiber. Tensile sample geometry is explained in Fig. 3. The displacement and applied loads recorded and the data were acquired digitally. The features of mixed elements are counted through a mixed strain gage/quarter-bridge strain gauge circuit provided from Tokyo (Sokki Kenkyuio Co., Ltd. The type of the used gage is a BFLA $-2-8$ with a gage obstruction of $120 \pm 0.3$ $\Omega$. At that point, the line was associated with a Data Acquisition (DAQ) connect framework for perusing the transverse strain.

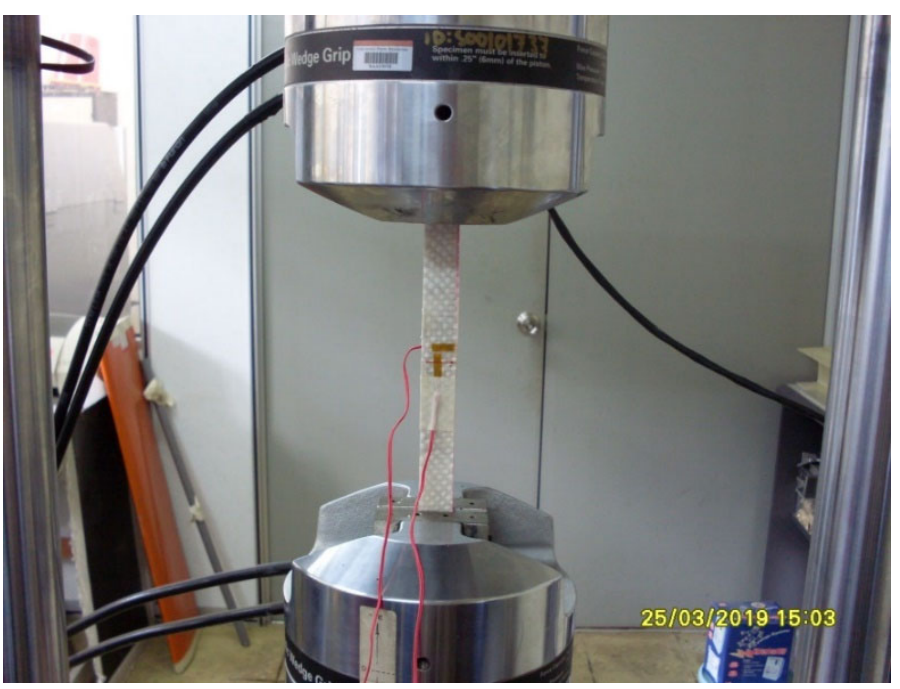

Fig. 3 Tensile Test device

\subsection{Coefficient of Thermal Expansion (CTE)}

An expansion in temperature causes the vibrational plentifulness of the atoms in the gem cross section of the strong increment. Expansion of thermal Coefficient, such as geometry is explained in Fig. 4. Along these lines the normal dispersing between the atoms builds, making the material extend. Perhaps, the temperature change, $\Delta \mathrm{T}$, is with the end goal that the material does not experience a stage change, at that point the coefficient of volumetric thermal expansion $\left(\alpha_{\mathrm{v}}\right)$ (Callister, 2000) of a material is characterized as

$\alpha_{v}=\frac{1}{V}\left(\frac{\partial \mathrm{V}}{\partial \mathrm{T}}\right)$

where $\mathrm{V}$ is the all-out volume of the material. It is thought that measurement acquired the Expansion of thermal Coefficient of linear $(\alpha l)$ as

$\alpha_{l}=\frac{1}{l}\left(\frac{\partial l}{\partial \mathrm{T}}\right)$

where $l$ is length

$\Delta l=l-l_{0}$

$\alpha_{l}=\frac{\Delta l}{l_{0}} \cdot \frac{1}{\Delta \mathrm{T}}$

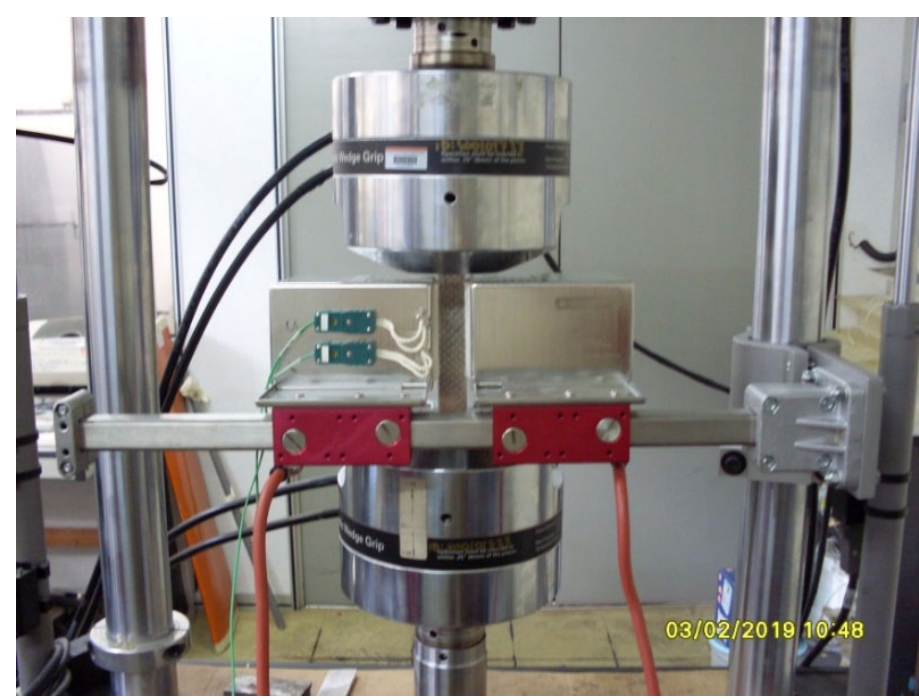

Fig. 4 find thermal expansion coefficient

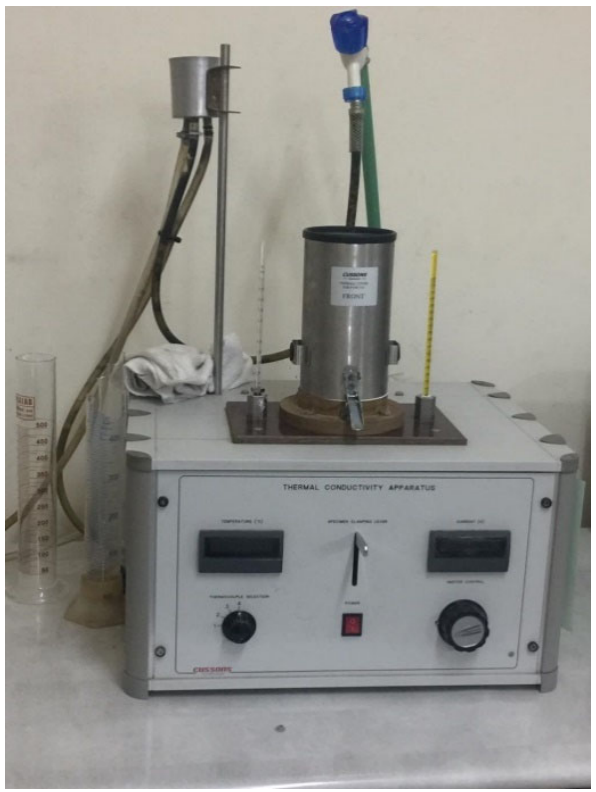

Fig. 5 Thermal conductivity Test device

\subsection{Thermal conductivity}


The thermal of conductivity $(\mathrm{k})$ of the composite material used for manufacturing heat sinks was measured by the apparatus shown in Fig. 5. The apparatus was manufactured by Cussons Technology Ltd. (England). The heat conducted was measured from the equation (Holman 1986):

$q=m C_{p} \Delta T$

$\dot{m}$ : Weight of collected water per time, $\mathrm{kg} / \mathrm{sec}$.

$\mathrm{C}_{\mathrm{p}}$ : Specific heat capacity, $\mathrm{J} / \mathrm{kg} . \mathrm{K}$.

$\Delta \mathrm{T}$ : Temperature between inlet water temperature and outlet water temperature, $(\mathrm{K})$. below:

Fourier Law is used to measure thermal conductivity (k) as shown

$$
q=-k A \frac{\Delta T}{\Delta X}
$$

where:

q: Heat flow (W) [measured from eq. (5)].

A; Area $\left(\mathrm{m}^{2}\right)$.

$\Delta \mathrm{T} / \Delta \mathrm{X}:$ Temperature gradient, and

$\mathrm{k}$ : Thermal conductivity.

\section{NUMERICAL MODEL}

This model of nanocomposite materials made in ANSYS version 15 Workbench programming. The composite material examples utilized for exploratory estimation of their thermal conductivities are as layer overlays. Each overlay is a MWCNT/Epoxy based fiber glass composite. Each spaceman was demonstrated utilizing the Design modeler highlight in ANSYS-workbench; their separate cross sections were produced and understood utilizing the enduring state thermal. Heat conduction through these nanocomposites materials is reproduced to foresee the matrix fiber interface thermal properties dependent on experimental esteems. To decrease the computational area, exertion, and time; a square formed unit cell was demonstrated which is an agent unit of the composite for example comprising of MWCNT/Epoxy with fiber encompassed by a framework yet having diverse volume division for MWCNT and material properties as the spacemen being demonstrated.

\subsection{Geometry}

To examine the transverse plane thermal conduction issue, a 3D model is made in the Design Modeler of ANSYS Workbench. The 3D model seems like the cross area of a nanocomposite cover with a framework (MWCNT/Epoxy) and fiber as appeared in Fig. 6.

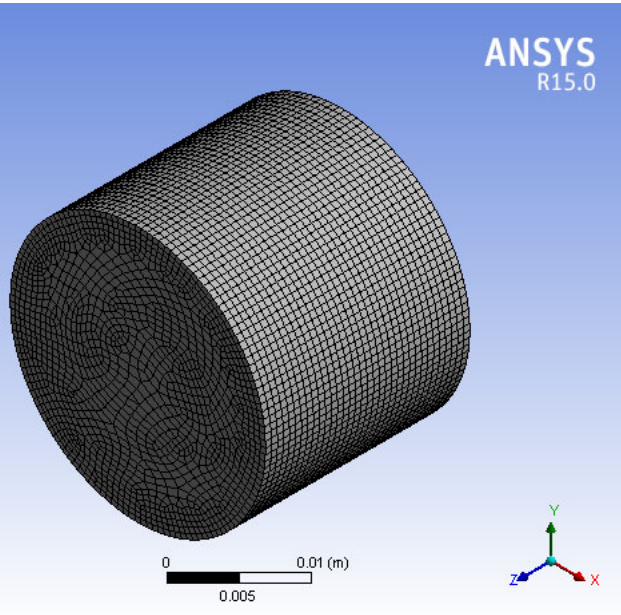

Fig. 6 Fine of Mesh spacemen

\section{RUSTLE AND DISCUSSION}

The values of the numerical model and the experimental work are listed. In this investigation a comprehensive study of mechanical and thermal properties of nanocomposite materials has been performed. The experimental results comprise the mechanical properties of the nanocomposites and matrix and thermal properties. The mechanical properties of MWCNT/epoxy and epoxy are calculated to be used for the delamination model used in the theory as shown in Table 4, while the mechanical properties of nanocomposites are measured to be used in the elastic deformation. Using the mechanical properties of the nanocomposite and matrix, the theoretical model was contrasted with the present experimental values was compared with a numerical solution by using program ANSYS version 15. the tensile test in woven fiber glass 20 plys, with a standard to measure the Poisson's ratio and lateral line. The influence of the mechanistic features of the MWCNT /epoxy exists on the general features of the nanocomposite ply. Increasing the features of the fiber leads to increasing in the influence as in the stress - strain for Fiber glass reinforce. It was demonstrated that the MWCNT / Epoxy gives higher properties but as well as give huge woven factors as shown in Table 5. The cause of this is the high features that it has.. The nanocomposite features are influenced in a direct way by the fiber glass features. This leads the highest features for the fiber glass components.

Table 4: The measured mechanical properties for MWCNT and Matrix epoxy

\begin{tabular}{|c|c|c|c|c|}
\hline Material & \multicolumn{2}{|c|}{ Tensile } & \multicolumn{2}{|c|}{ Compression } \\
\hline Epoxy & $\begin{array}{c}\sigma_{\text {ut }} \\
(\mathbf{M P a}) \\
57.6\end{array}$ & $\begin{array}{c}\mathbf{E} \\
(\mathbf{G P a}) \\
1.714\end{array}$ & 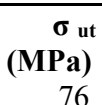 & $\begin{array}{c}\mathbf{E} \\
\text { (GPa) } \\
1875\end{array}$ \\
\hline $\begin{array}{c}\text { 2\% MWCNT } \\
+ \text { Epoxy }\end{array}$ & 42 & 5.6 & 52 & 2.1 \\
\hline
\end{tabular}

Table 5: The mechanical properties for the nanocomposites manufactured

\begin{tabular}{lllllll}
\hline \multicolumn{3}{c}{ Composition } & \multicolumn{3}{c}{ Woven composite ply } \\
\hline Fiber & Powder & Matrix & Vol\% & Tensile & Compression \\
\hline FG & MWCNT & Epoxy & & $\begin{array}{l}\sigma_{\text {ut }} \\
(\mathrm{MPa})\end{array}$ & $\begin{array}{l}\text { E } \\
(\mathrm{GPa})\end{array}$ & $\begin{array}{l}\sigma_{\mathrm{ut}} \\
(\mathrm{MPa})\end{array}$ \\
\hline FG & MWCNT & Epoxy & $1 \%$ & 132.2 & 12.1 & 58.47 \\
\hline FG & MWCNT & Epoxy & $2 \%$ & 145.42 & 13.3 & 65.79 \\
\hline FG & MWCNT & Epoxy & $4 \%$ & 151.66 & 14.5 & 67.38 \\
\hline FG & MWCNT & Epoxy & $6 \%$ & 157.34 & 15.91 & 69.57 \\
\hline FG & MWCNT & Epoxy & $8 \%$ & 168.65 & 16.87 & 67.44 \\
\hline FG & MWCNT & Epoxy & $10 \%$ & 177.98 & 17.54 & 66.68 \\
\hline & & & & & &
\end{tabular}

In the thermal conductivity, the experimental of composite material of multi-wall carbon nanotube (MWCNT)/Epoxy and reinforced fiberglass for information that the thermal conductivity of epoxy is low value $0.1525 \mathrm{~W} / \mathrm{m}{ }^{\circ} \mathrm{C}$ so that the thermal conductivity of the fiber glass $1.35 \mathrm{~W} / \mathrm{m}^{\circ} \mathrm{C}$ compared to the thermal conductivity of a very high multi - wall carbon nanotube (MWCNT) is $3000 \mathrm{~W} / \mathrm{m}^{\circ} \mathrm{C}$ where thermal conductivity dependent on the type of particles used and on its position during the middle. The thermal conductivity of the composite materials fiber glass MWCNT/Epoxy is increased with the increasing of the volume fraction of the MWCNT as shown in Table 6. The thermal conductivity value was $6.52 \mathrm{~W} / \mathrm{m}{ }^{\circ} \mathrm{C}$ at volume fraction $10 \%$ at the temperature of $25^{\circ} \mathrm{C}-71.012{ }^{\circ} \mathrm{C}$ as shown in Fig. 7.

Results obtained from experimental of thermal conductivity measurements conducted on fiber glass MWCNT/Epoxy composite material are shown in Fig. 7 the manufacturing of multi wall carbon nanotube /epoxy composites different volume fraction from $1 \%$ to $10 \%$ with an increment of $2 \%$ therefor thermal conductivity increased. The effect of the volume fraction at the expansion thermal coefficient of fiber glass MWCNT/Epoxy of the nanocomposite material, which was carried out on different samples, the volume fraction $(1-10) \%$, where the thermal expansion coefficient was $5.7 \times 10^{-5}\left(1 /{ }^{\circ} \mathrm{C}\right)$ at the volume fraction 
$10 \%$ at the temperature of $25^{\circ} \mathrm{C}-71.012^{\circ} \mathrm{C}$ as shown in Fig. 8. The results decreased thermal expansion coefficient by increasing the volume fraction of MWCNT, which explains the increase of nanoparticles to prevent expansion. The mesh independent size different (medium and fine) as shown in the Figs. 9-20 distribution of temperature in the composite material fiber glass MWCNT/Epoxy after adding the mechanical properties and thermal properties under effect of heat flux $15000 \mathrm{~W} / \mathrm{m}^{2}$, thermal conductivity in the simulation program ANSYS as shown figures the distribution of the temperature of the different volume fractions of each specimen The finite element by using ANSYS is good agreement with experimental data for different volume fraction as shown in the Table 7.

Table 6: The thermal properties for the nanocomposites manufactured

\begin{tabular}{cccc}
\hline \multirow{2}{*}{$\begin{array}{c}\text { Nanocomposite } \\
\text { Materials }\end{array}$} & $\begin{array}{c}\text { MWCNT } \\
\text { Vol \% }\end{array}$ & $\begin{array}{c}\text { Thermal } \\
\text { conductivity } \\
(\mathrm{W} / \mathrm{m} \text { C })\end{array}$ & $\begin{array}{c}\text { Thermal } \\
\text { expansion } \\
\text { coefficient } \\
(1 / \mathrm{C})\end{array}$ \\
\hline FG+ MWCNT/Epoxy & $1 \%$ & 4.21 & $9.8\left(10^{-5}\right)$ \\
\hline FG+ MWCNT/Epoxy & $2 \%$ & 4.64 & $9\left(10^{-5}\right)$ \\
\hline FG+ MWCNT/Epoxy & $4 \%$ & 5.13 & $8.1\left(10^{-5}\right)$ \\
\hline FG+ MWCNT/Epoxy & $6 \%$ & 5.56 & $7.3\left(10^{-5}\right)$ \\
\hline FG+ MWCNT/Epoxy & $8 \%$ & 6.1 & $6.62\left(10^{-5}\right)$ \\
\hline FG+ MWCNT/Epoxy & $10 \%$ & 6.52 & $5.7\left(10^{-5}\right)$ \\
\hline
\end{tabular}

Table 7: The compared temperature between experimental data with ANSYS program data for the nanocomposites manufactured

\begin{tabular}{lccccc}
\hline \multirow{2}{*}{$\begin{array}{c}\text { Nanocomposite } \\
\text { Materials }\end{array}$} & $\begin{array}{c}\text { MWCNT } \\
\text { Vol \% }\end{array}$ & \multicolumn{2}{c}{$\begin{array}{c}\text { Experimental } \\
\text { Data }\end{array}$} & \multicolumn{2}{c}{ ANSYS Data } \\
\cline { 3 - 6 } & & $\mathrm{T}_{\mathrm{m}}$ & $\mathrm{T}_{\max }$ & $\mathrm{T}_{\mathrm{m}}$ & $\mathrm{T}_{\max }$ \\
\hline FG+ MWCNT/Epoxy & $1 \%$ & 58.23 & 91.64 & 60.63 & 96.341 \\
\hline FG+ MWCNT/Epoxy & $2 \%$ & 55.42 & 86.47 & 57.327 & 89.655 \\
\hline FG+ MWCNT/Epoxy & $4 \%$ & 52.12 & 81.3 & 54.24 & 83.48 \\
\hline FG+ MWCNT/Epoxy & $6 \%$ & 49.9 & 74.8 & 51.978 & 78.957 \\
\hline FG+ MWCNT/Epoxy & $8 \%$ & 47.7 & 70.56 & 49.59 & 74.18 \\
\hline FG+ MWCNT/Epoxy & $10 \%$ & 46.6 & 69.17 & 48.006 & 71.012 \\
\hline
\end{tabular}

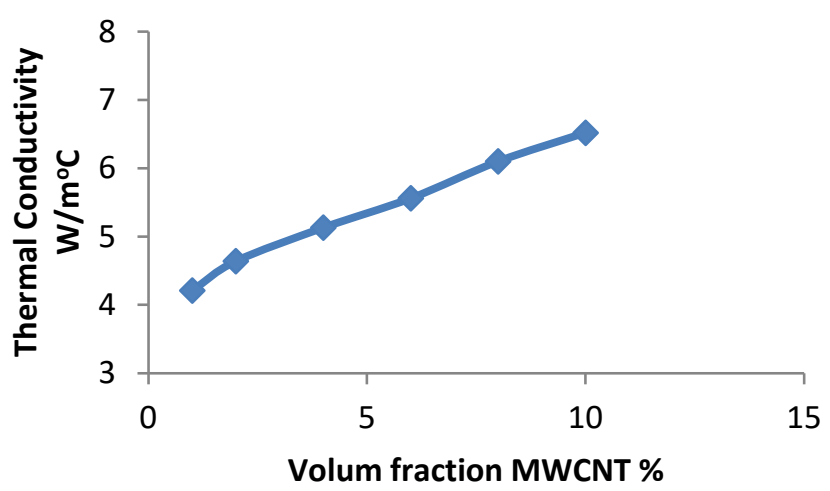

Fig. 7 Thermal conductivity of nanocomposite materials Fiber glass MWCNT / Epoxy with the volume fraction of MWCNT

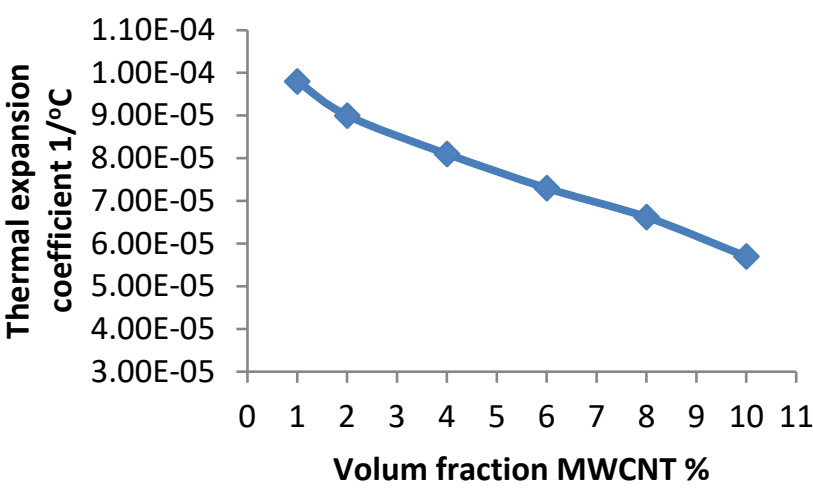

Fig. 8 Thermal expansion coefficient of nanocomposite materials Fiber glass MWCNT / Epoxy with volume fraction of MWCNT

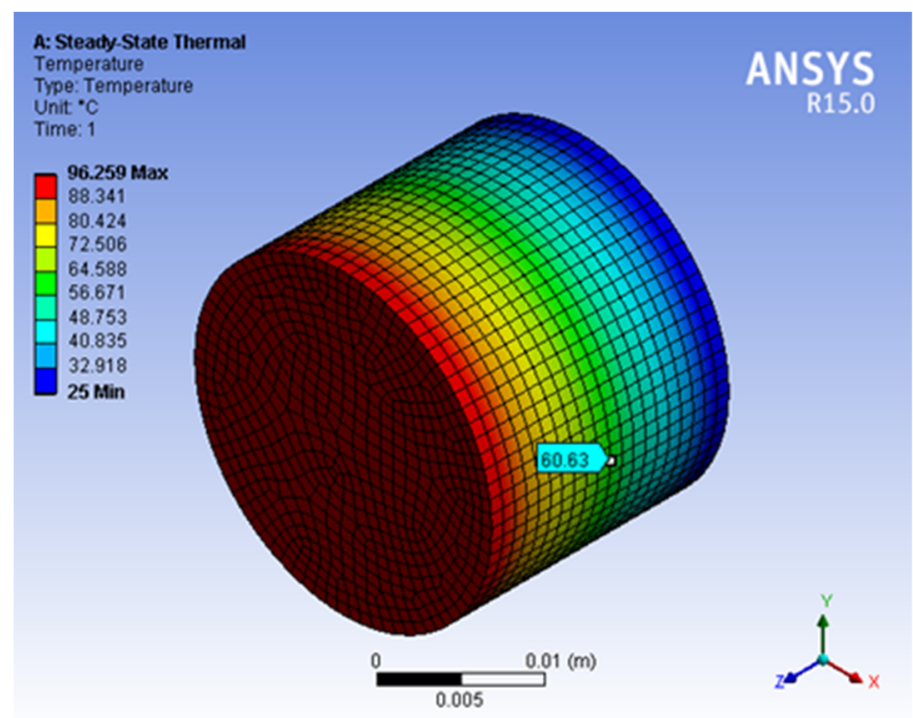

Fig. 9 Medium mesh 1\% MWCNT

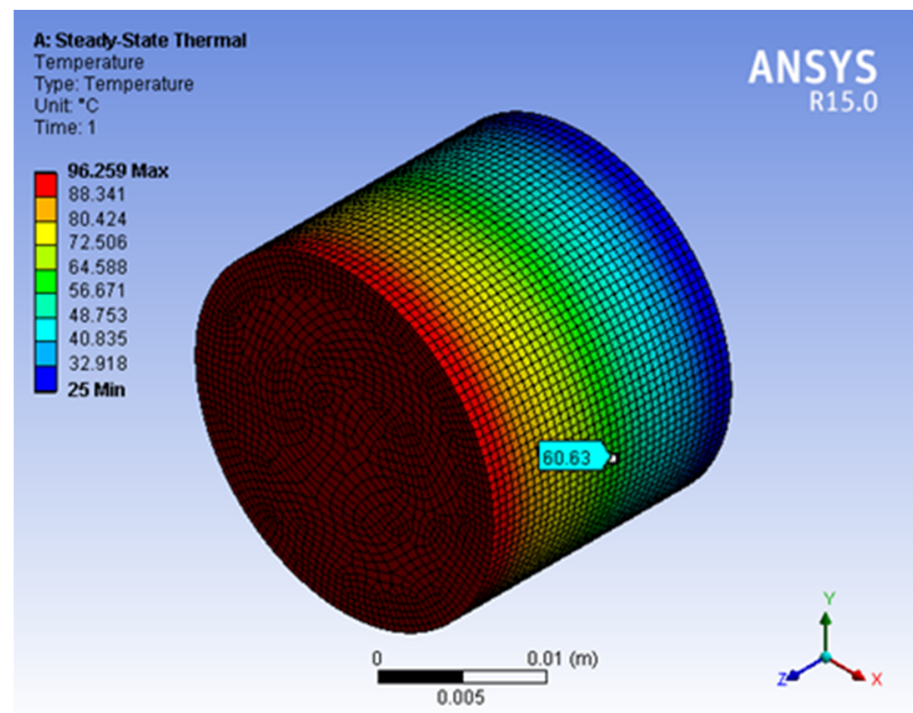

Fig. 10 Fine mesh 1\% MWCNT 


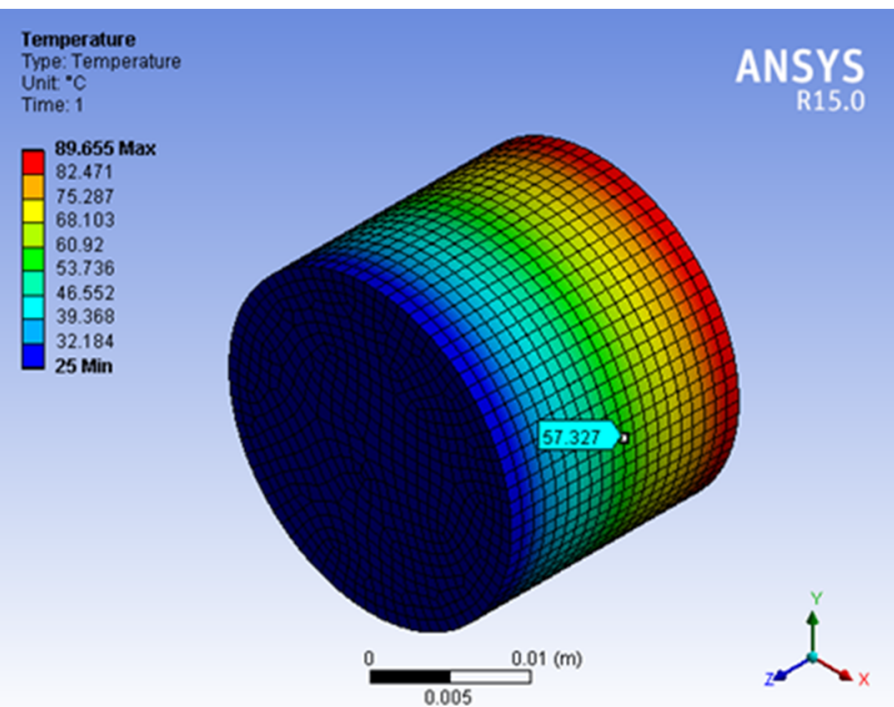

Fig. 11 Medium mesh 2\% MWCNT

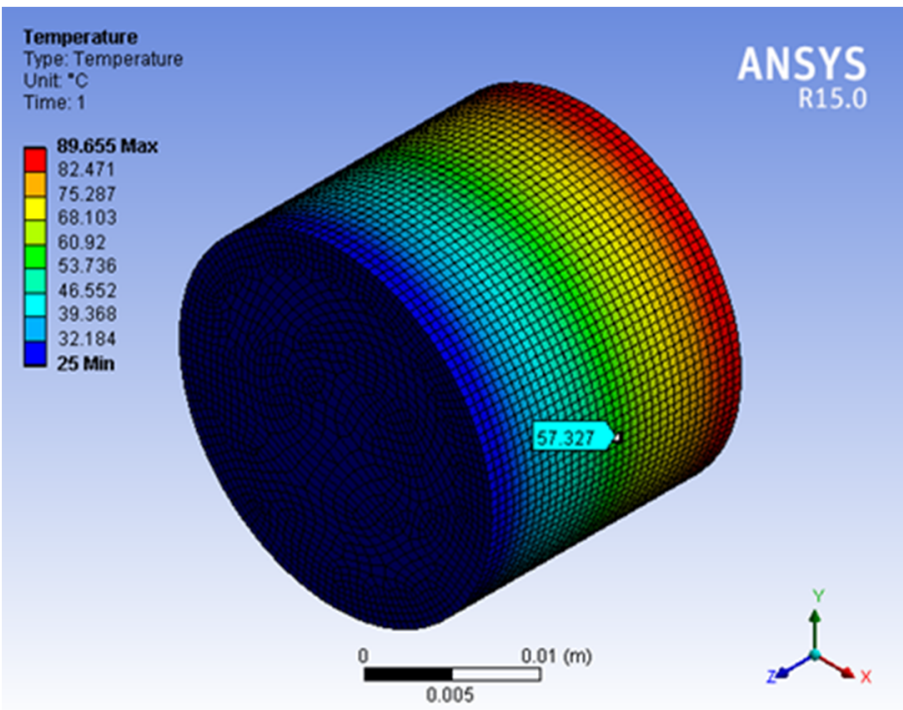

Fig. 12 Fine mesh $2 \%$ MWCNT

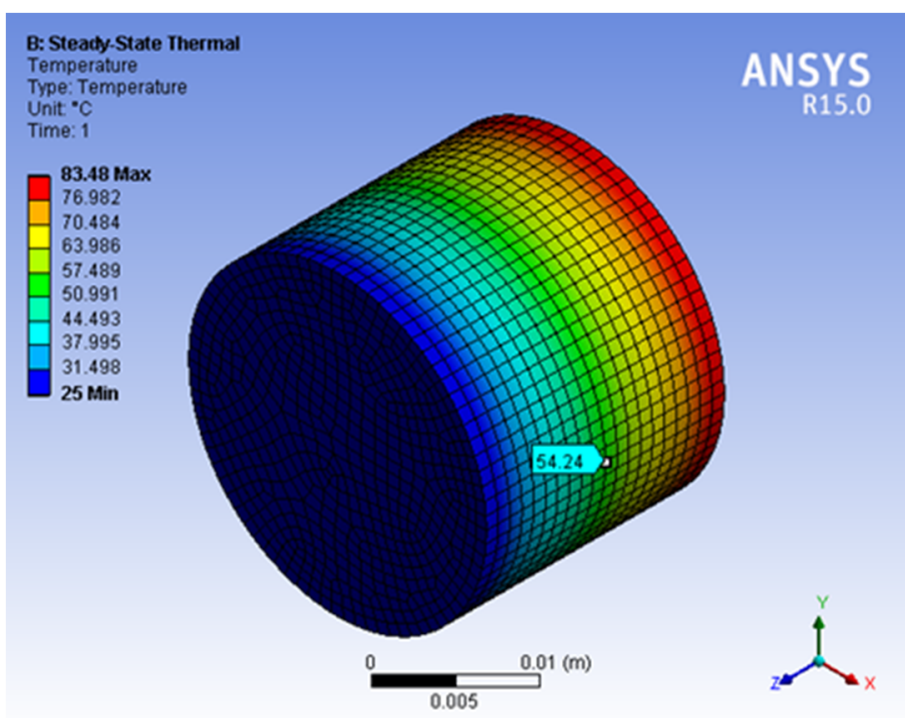

Fig. 13 Medium mesh 4\% MWCNT

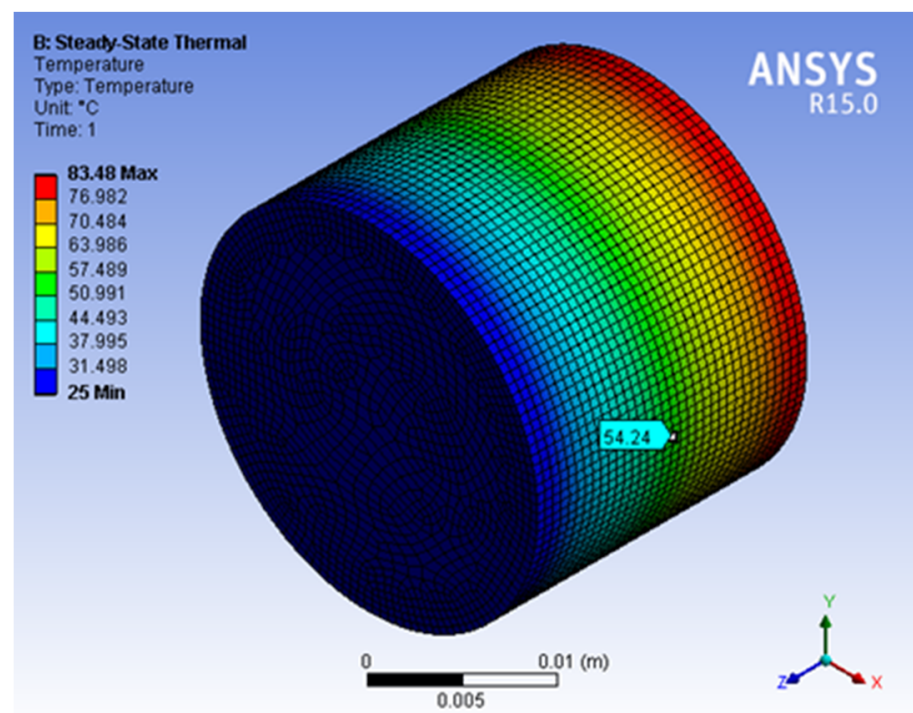

Fig. 14 Fine mesh 4\% MWCNT

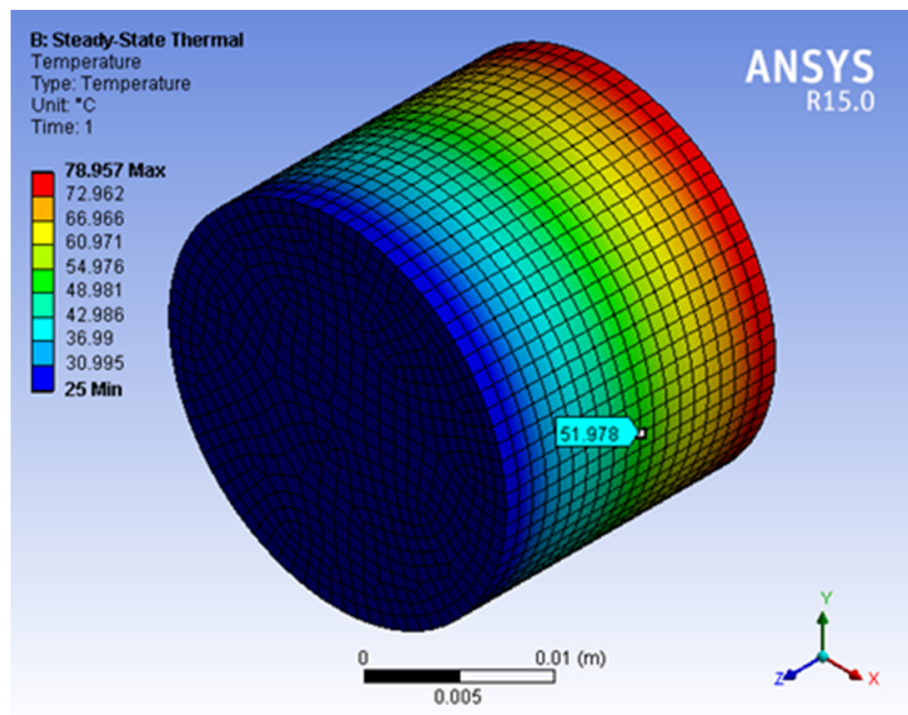

Fig. 15 Medium mesh 6\% MWCNT

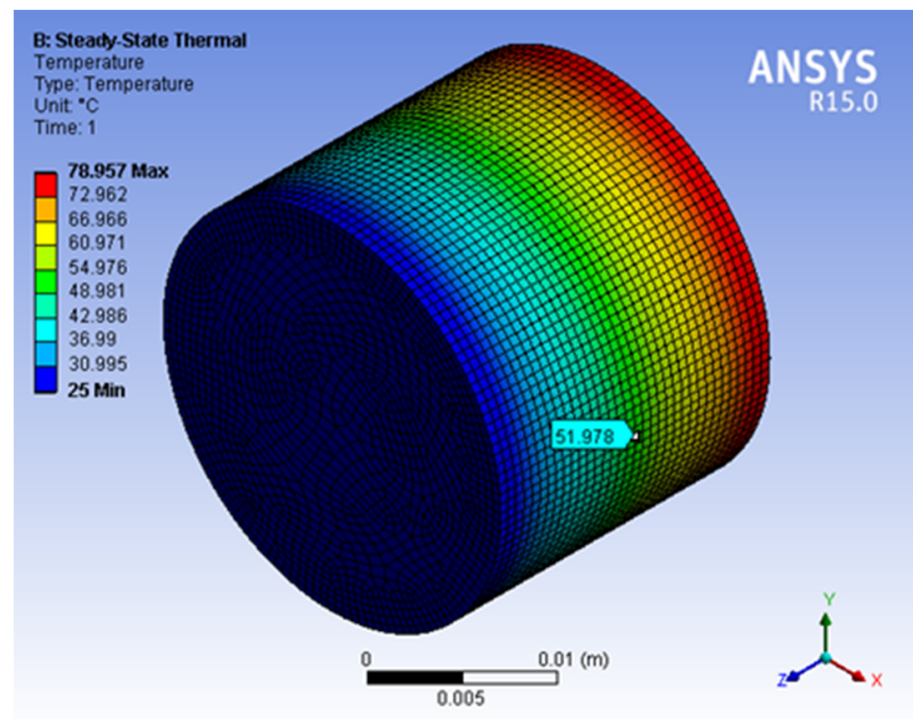

Fig. 16 Fine mesh 6\% MWCNT 


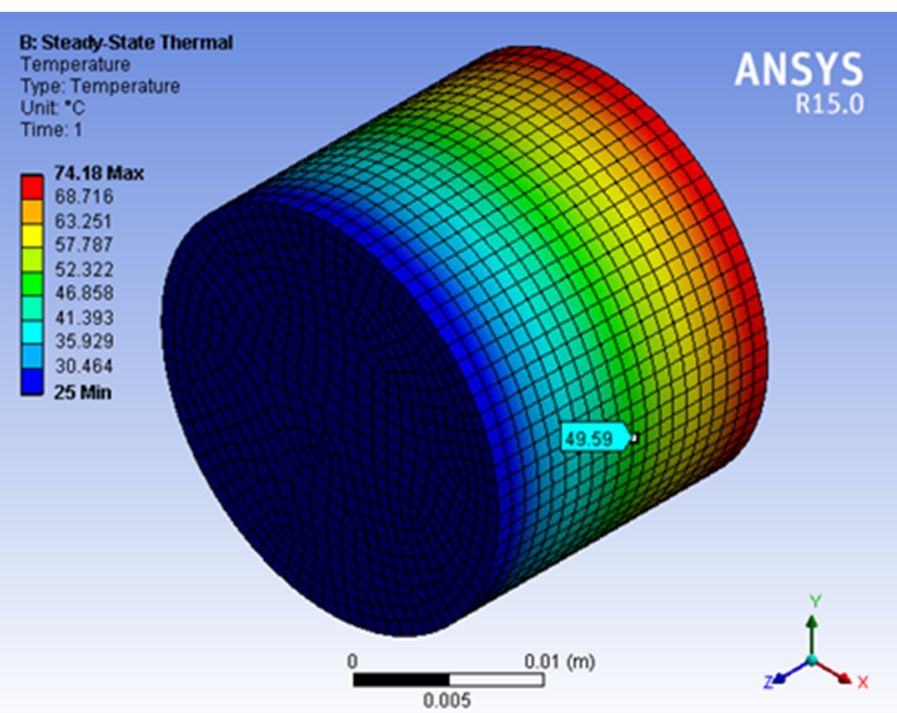

Fig. 17 Medium mesh $8 \%$ MWCNT

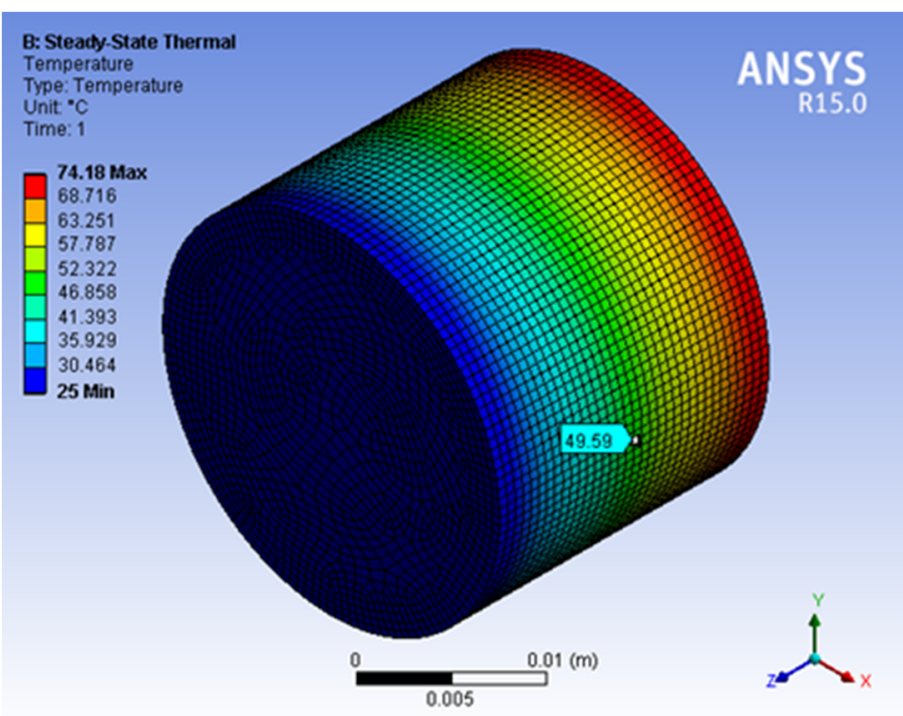

Fig. 18 Fine mesh $8 \%$ MWCNT

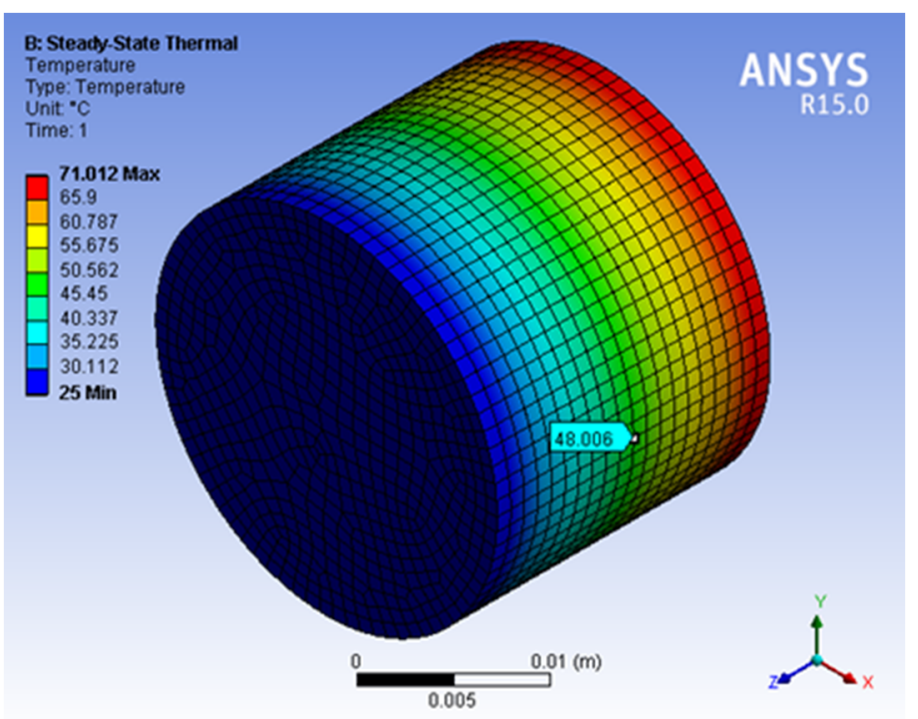

Fig. 19 Medium mesh 10\% MWCNT

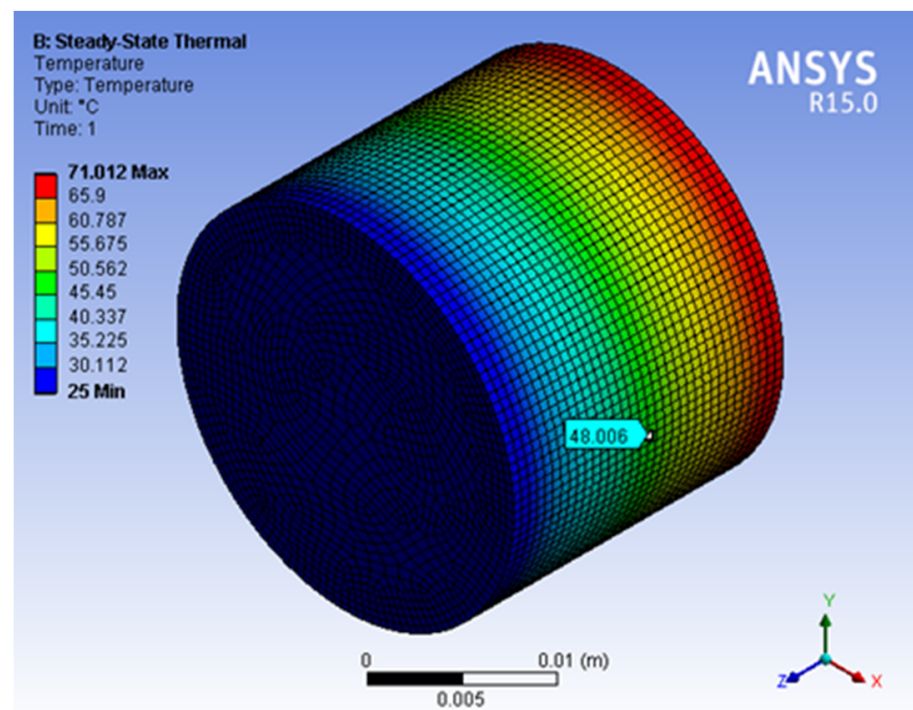

Fig. 20 Fine mesh 10\% MWCNT

\section{CONCLUSIONS}

According to the results Numerical and experimental works that was done out in this investigation, the primary conclusions that can be drawn are:

1. To Design and fabrication of composite plate specimens for different volume fractions for fibers glass woven and multi wall carbon nanotube MWCNT/epoxy and test specimens.

2. From the tests for determining the mechanical properties of the composite materials (Fiber Glass + MWCNT / Epoxy) it was found that these properties for woven fiber glass composite were increased for different volume fraction MWCNT.

3. From the results it is increase of the volume fraction MWCNT increase the thermal conductivity and decreased the thermal expansion coefficient.

4. The enhancement of the temperature distribution is experimentally and compared with the finite element by using ANSYS is good agreement data for different volume fraction of nanocomposite materials (Fiber Glass + MWCNT / Epoxy)

\section{NOMENCLATURE}

$T \quad$ Temperature (C)

$\alpha_{v} \quad$ coefficient of volumetric thermal expansion $(\mathrm{J} / \mathrm{kg} \cdot \mathrm{K})$

$\alpha_{l} \quad$ Expansion of thermal Coefficient of linear $(\mathrm{J} / \mathrm{kg})$

$k \quad$ thermal conductivity $(\mathrm{W} / \mathrm{m} \cdot \mathrm{K})$

$l \quad$ length $(\mathrm{m})$

$q \quad$ heat flow (W)

$\dot{\mathrm{m}} \quad$ Weight of collected water per time, $\mathrm{kg} / \mathrm{sec}$

A Area $\left(\mathrm{m}^{2}\right)$.

$C_{p} \quad$ Specific heat capacity, (J /kg.K)

$F G \quad$ Fiber glass

\section{REFERENCES}

Ajayan PM, Ebbesen TW, 1997, "Nanometer-size Tubes of Carbon," Rep. Prog. Phys., 60, $1025-1062$.

https://doi.org/10.1088/0034-4885/60/10/001

Ahmad, B., Iqbal, Z., 2017, "An Effect of Cattaneo Christov Heat Flux Model for Eyring Powell Fluid over an Exponentially Stretching Sheet," Frontiers in Heat and Mass Transfer, 8, 22 https://doi.org/10.5098/hmt.8.22 
Callister, W.D., 2000, Materials Science and Engineering - An Introduction, 5th edition, Wiley and Sons Inc.

https://doi.org/10.1108/acmm.2000.12847aae.001

Ebbesen TW, Ajayan PM., 1992, "Large-scale Synthesis of Carbon Nanotubes," Nature, 358, 220 -222. https://doi.org/10.1038/358220a0

Habib-Olah Sayehvand, Shirley Abelman, Amir Basiri Parsa, 2017, "MHD Nanofluid Flow With Viscous Dissipation And Joule Heating Through A Permeable Channel," Frontiers in Heat and Mass Transfer, 9, 30. https://doi.org/10.5098/hmt.9.30

Holman, J.P, Heat Transfer, sixth edition, Mc Graw-Hill, Inc. New York, 1986

Iijima S., 1991, "Helical Microtubules of Graphitic Carbon," Nature, 354: 56 - 58. https://doi.org/10.1038/3540560

Nandeppanavara, M.M., and Shakunthala, S., 2017, "Flow and Heat Transfer Of Carbon Nanofluids Over A Vertical Plate," Frontiers in Heat and Mass Transfer, 9, 27. https://doi.org/10.5098/hmt.9.27

Tashkandi, M.A., and Aydi, A., 2018, "Heat Transfer Intensification in A 3D Cavity Using Hybrid Cnt- $\mathrm{Al}_{2} \mathrm{O}_{3}$ (15-85\%) Nanofluid," Frontiers in Heat and Mass Transfer, 11, 27 https://doi.org/10.5098/hmt.11.27
Okutan, B., 2002, "Effects of Geometric Parameters on the Failure Strength for Pin-loaded Multi-loaded Multi-directional Fiber-glass Reinforced Epoxy Laminate," Composites Part B: Engineering, 33, 56757. https://doi.org/10.1016/S1359-8368(02)00054-9

Tacir, IH, Kama, JD Zortuk, M Eskimez S, 2006, "Flexural properties of glass fibre reinforced acrylic resin Polymers," Australian Dental Journal, 16(2), 265-275

https://doi.org/10.1111/j.1834-7819.2006.tb00401.x

Uleiwi, J.K., 2007, "Experimental Study of Flexural Strength of Laminate Composite Material," Eng. \& Technology, 25(Suppl. 3), 454466.

Wen, H.W., Reddy, T. Y., Reid, S. R. and Soden, P. D., 1998, "Indentation, Penetration and Perforation of Composite Laminates and Sandwich Panels under Quasi-Static \& Projectile Loading," Key Engineering Materials, 141-143, 501-552.

https://doi.org/10.4028/www.scientific.net/KEM.141-143.501 